\title{
Effect of Dynamic Vents on the Thermal Comfort of a Passenger Car
}

\author{
Om Prakash Singh1, ${ }^{*}$ - Rohit Raut ${ }^{1}$ - Mrinmoy Biswas ${ }^{1}$ - Ramji Singh ${ }^{2}$ \\ ${ }^{1}$ Indian Institute of Technology Mandi, School of Engineering, India \\ 2 Aryabhata Knowledge University, India
}

\begin{abstract}
When a vehicle is parked under the direct sun, heat flux entering the cabin through the roof top becomes higher and thus, sets up high temperature gradients beneath the roof. Dynamic airvents are provided to cool the cabin when the air-conditioner is switched on. Unfortunately, the car manufacturer does not specify any guidelines for the driver/passengers regarding the use of dynamic airvents in cars. This study aims to investigate the effect of dynamic vents on the cabin temperature when cooling is initiated. Computational fluid dynamics (CFD) analysis was performed in three stages. In the first stage, temperature data were measured experimentally at various locations in the car and at different times of the day. In the second stage, a 3D CAD model of the same car was generated by measuring the design data. In third stage, a CFD model of the car was developed and used to validate the experimental data. Simulations were performed to study the effect of dynamic vents. The results shows that faster cooling of the cabin and maintaining a uniform temperature distribution inside the cabin is possible at a particular vent angle, which the manufacturer does not specify or indicate for the vehicle. These results suggest an energy saving potential without any changes in the air-conditioning unit itself.
\end{abstract}

Keywords: thermal comfort, dynamic vents, computational fluid dynamics, HVAC, cabin temperature

Highlights

- Effect of dynamic airvents on car cabin temperatures are reported.

- $\quad$ CFD model is validated against experimentally measured data during the daytime.

- Uniform temperature distribution during the when cooling is initiated is observed for a particular vent angle.

- $\quad$ For the first 3 to 4 minutes the car air-conditioner should be operated at a high flow setting using the dynamic vents.

- $\quad$ Results can be utilized to save energy without any changes in the air-conditioning unit.

\section{INTRODUCTION}

Environment friendliness and energy efficiency are the mantra of today's world in each and every sector of development. The automotive industry is also now switching to zero emission car concepts to be in congruence with this concept. During this transformation, however, consumer satisfaction should not be hampered. The consumer is satisfied if he feels comfortable using a given commodity. Comfort, being a physiological or psychological factor, is basically a subjective term. Thermal comfort is therefore also a subjective term, dependent on heat conduction, convection, radiation and evaporative heat loss. Thermal comfort is the condition of mind that expresses satisfaction with the thermal environment and is assessed by subjective evaluation (ANSI/ ASHRAE Standard 55) [1]. The predicted mean vote (PVM) is the most recognized thermal comfort model whose seven point scale varies from -3 (cold) to +3 (hot). Zero is the ideal value, representing thermal neutrality, i.e. a neutral thermal sensation is achieved when the human body is in thermal equilibrium with the surroundings. Heat generated by a human body depends on the metabolism of the system. Each part of human body has a different metabolism rate. Because of the numerous differences between the physiological and psychological satisfaction of human beings it is very difficult to pinpoint a temperature that would provide the same level of thermal comfort to every passenger of a vehicle. The thermal comfort for the passenger of the car is maintained by the HVAC (Heating, Ventilation and Air-Conditioning) system. Therefore, the number of vents, air-flow temperature and velocity, cabin temperature and relative humidity are the main parameters that control the inner environment of a passenger car. The thermal comfort sensation is optimal when the relative humidity value is about $50 \%$. Humidity fluctuations play a minor role if the values are in the range of $30 \%$ to $70 \%$ [2]. Thermal comfort in the vehicle is achieved (i) by ensuring temperatures of $20{ }^{\circ} \mathrm{C}$ to $22{ }^{\circ} \mathrm{C}$, as a result of air temperature, delimitation areas, humidity and air velocity in accordance with the activity level and clothing insulation of the occupants, (ii) by avoiding situations such as the occupants coming into contact with very cold or very hot surfaces, (iii) by avoiding air 
currents. These requirements must be met throughout the entire year, both in summer and wintertime [2].

Much research has been carried out to enhance the thermal comfort inside the passenger car. Chen et al. [3] in their research suggested an energy efficient way of delivering comfort to an occupant in the car. Their research focused on the design and CFD analysis of an energy efficient HVAC system with spot cooling by strategically placing multiple nozzles in the vehicle directed at specific body parts. In their experiment the nozzle design and nozzle locations were paramount to deliver and achieve energy efficiency. In another research paper on the design of dynamic air vents and airflow analysis in a passenger car cabin by Varad et al. [4] they suggested the concept of dynamic vents. They analyzed the effect of dynamic vents and compared them with the steady air vent model. The results projected a considerable drop in the average cabin temperature. They also noted various effects of dynamic air vents. Walgama et al. [5] in their paper classified the work with respect to the passenger compartment environment or the condition of the passengers and their interaction with the compartment. The effects of factors such as flow field and temperature field were taken into consideration. They proposed computational and empirical models for forecasting thermal comfort in the non-uniform transient environment of the passenger car. Studies of air-flow and temperature fields inside a passenger compartment for improving thermal comfort and saving energy was investigated by Zhang et al. [6]. They investigated the influence of different factors on thermal comfort and energy consumption. Temperature distributions and the flow field inside a passenger car were simulated and analyzed with and without passengers. Their simulation results revealed various factors that ameliorate the thermal comfort and energy consumption such as the significance of air inlet temperature and the external conditions. The results also showed that the number of passengers also affects the conditions. The window materials also influence the thermal insulation of the vehicle. To improve the uniformity of the temperature field around the driver's foot zone a better flow circulation near the compartment bottom is favorable, for example, Ruzic [7].

Ruzic [7] used the method of localized distribution of air to improve thermal comfort. Alexandrov et al. [8] used CFD methods to investigate the flow patterns inside the car cabin. One interesting study by Wan and Kooi [9] was that they investigated the position of supply and exhaust opening on comfort in a passenger car. They showed that optimizing the location of the exhaust system maximizes thermal comfort. There have been numerous studies in the recent past on thermal comfort using CFD. Recently, Zval et al. [10] presented a three-dimensional adiabatic flow simulation in an air-conditioned vehicle passenger compartment and proposed a combination of turbulence models and near wall treatment that can be used for simulations of thermal comfort and determination of comfort of driver and passengers. They performed CFD modeling using models such as eddy - viscosity standard, RNG k- $\varepsilon$ and SST k- $\omega$ models. Although the thermal comfort of vehicles has been studied extensively, Ismail et al. [11] went a step further to investigate the thermal comfort assessment of workers in automotive industries. They reported that most of the workstations in the automotive industry were uncomfortable.

The greenhouse effect in passenger cars happens when the greenhouse gases trapped inside a passenger car absorb the thermal radiation from the sun and reradiate it inside in all direction, thus increasing the cabin temperature. This effect is prominent when we park the car for few hours in direct sunlight with all the windows closed. There is a considerable difference in the external ambient temperature and the temperature inside the cabin. In order to overcome this issue a precooling system such as a solar powered pre-cooling ventilation system on the roof or remote-activated air-conditioning for pre-cooling the cabin has to be embedded so that the car cabin can be cooled before the passenger enters the car. However, the problem with this system is the high cost. A high additional cost will have to be borne by the customer for installing this system. A completely new system also has to be placed in the car to incorporate the additional unit. In today's world where cars are becoming more and more optimized, incorporating a new technology for cooling is not efficient and increases the weight of the overall system.

The aim of this research is to find a solution to this problem without too many changes to the current air-conditioning system. The car manufacturers do not specify the vane angle and air speed so that faster cooling can be initially achieved. Energy could also be saved since a particular vane angle cools only a certain part of the cabin space, which may or may not be useful. We have investigated the effect of vane direction and inbuilt speed to find the optimal angle and speed to maximize the cooling as quickly as possible. We have also incorporated the system of dynamic vents and analyzed its effect on the rate of cooling and average cabin temperature. The speed of oscillation of the vents has also been investigated. 


\section{CFD MODEL}

\subsection{Computational Domain and Boundary Conditions}

The computational domain incorporates the space inside the cabin including the front and back seats. The inner surfaces of the passenger cabin including the top surface, the sidewall and the dashboard region constitute the boundary of the computational domain. The cabin design is of a typical passenger vehicle (in this case a hatchback, Maruti Suzuki Alto 800). The $z=0$ plane passes through the center of the cabin space dividing the space into two symmetrical halves. Two separate armchairs are there on each side of $z=0$ plane in the front area, whereas a long bench at the rear sheet serves for the rear passengers.

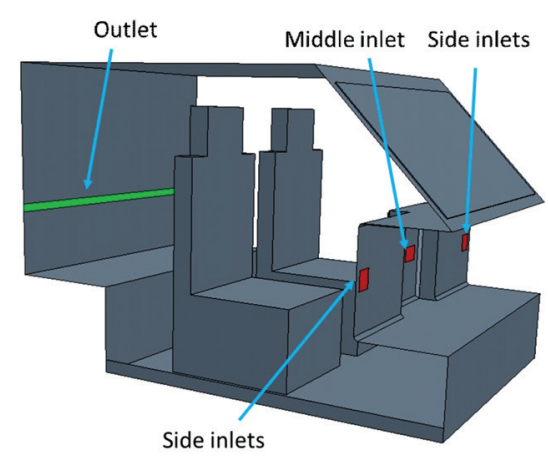

Fig. 1. Airflow inlets and outlets of the cabin space

The air-inlets are present on the dashboard as shown in Fig. 1, two inlets at the center of the dashboard, which will be referred as center inlets and two air-inlets one on each side of the driver and passenger near the front windows. All inlets are horizontally aligned, while air outlet is positioned on the rear wall. This is an assumed exit since the air-conditioning works in outside-circulation mode. The boundaries, i.e. the top surface, sidewalls, back wall, doors and windows, are treated as solid walls. The cabin is assumed to be sealed from the inside thus eliminating additional air flow interaction. The flow condition is assumed to be incompressible with constant thermo-physical properties of air. The gravity is defined in the computational domain in the negative $Y$ direction i.e. downward direction. The air inlet velocity is different for two different cases, one medium-flow and one high-flow. In the medium flow case, the specification of the air velocity is $4.5 \mathrm{~m} / \mathrm{s}$ for the center inlets and $2 \mathrm{~m} / \mathrm{s}$ for the side inlets. While in the high flow case, the air-inlet velocity is set at 6.3 $\mathrm{m} / \mathrm{s}$ for the center inlets and $3 \mathrm{~m} / \mathrm{s}$ for the side inlets.
The turbulent intensity was set at $10 \%$ everywhere and a flow-split outlet was set as the airoutlet boundary condition. The walls of the cabin also serve as a different type of boundary condition as these are the interface between the interior of the cabin and the outside ambient conditions. Solar heat flux enters the cabin from all the windows, the total radiation entering depends on the angle of incidence $\alpha$. To calculate the incident radiation heat flux density, we used the equation: $P=\varepsilon \sigma A T^{4}$, where $\varepsilon$ is the surface emissivity, $\sigma$ is the Stefan-Boltzmann constant $\left(5.67 \times 10^{-8} \mathrm{~W} /\left(\mathrm{m}^{2} \cdot \mathrm{K}^{4}\right)\right), T$ is the surface temperature in Kelvin and $A$ is the surface area. The car surface was coated with white paint whose emissivity is 0.9 , the total projected surface area was $1.92 \mathrm{~m}^{2}$ and the surface temperature was $318^{\circ} \mathrm{K}$. Using the above equation, the solar irradiation comes out be $1000 \mathrm{~W} / \mathrm{m}^{2}$. For surfaces like the top bottom surface windows, windscreens and side walls, the following equation based on Newton's law is used to calculate the heat flux due to solar radiation [12],

$$
\begin{gathered}
q=h_{0}\left(t_{0}-t_{w}\right)+\alpha G, \\
q=h_{0}\left[\left(t_{0}+\frac{\alpha G}{h_{0}}\right)-t_{w}\right]=h_{0}\left(t_{z}-t_{w}\right), \\
t_{z}=t_{0}+G / h_{0},
\end{gathered}
$$

where $q$ is a heat flux $\left[\mathrm{W} / \mathrm{m}^{2}\right] ; h_{0}$ convective heat transfer coefficient $\left[\mathrm{W} /\left(\mathrm{m}^{2} \cdot \mathrm{K}\right)\right] ; \quad t_{0}$ outside air temperature $\left[{ }^{\circ} \mathrm{C}\right] ; t_{w}$ outside surface temperature of the cabin $\left[{ }^{\circ} \mathrm{C}\right] ; \alpha$ absorptivity of sun radiation; $G$ incident radiation heat flux density (solar irradiation) $\left[\mathrm{W} / \mathrm{m}^{2}\right] ; t_{z}$ synthetic temperature $\left[{ }^{\circ} \mathrm{C}\right]$. The synthetic temperature is derived to ease the process of calculating solar heat flux using Newton's law of cooling as shown in Eq. (1). The convective heat transfer coefficient is calculated as follows:

$$
h_{0}=1.163(4+12 \sqrt{v}) \text {, }
$$

where $v$ is the wind speed relative to the vehicle in $\mathrm{m} / \mathrm{s}$. The calculated $h_{0}$ was $31 \mathrm{~W} / \mathrm{m}^{2} \mathrm{~K}$ with a standard deviation of 1.51 based on the ambient velocity variation.

The window glass is transparent to most solar radiation. The radiation effect is neglected in the simulation and hence, from the available data of the radiation intensity, the area of the window and the incident angle of solar radiation, the calculated heat flux is assumed to enter from the top surface. The seat boundaries are set to flow obstacles with zero heat flux, i.e. they do not contribute to the heat entering into the cabin. 


\subsection{Grid Generation and Numerical Methods}

Unstructured grids with tetrahedron elements have been adopted, with the size of elements given as $1 \mathrm{~cm}$ except for the air inlets and outlets and the top surface. The air-inlets and outlets are refined locally with an element size of $1 \mathrm{~mm}$ since a larger temperature and velocity gradient is present at these locations. At the top surface the element size is $6 \mathrm{~mm}$ since a constant solar heat flux is entering the cabin space from the top surface. The total numbers of control volumes present in the computational domain are 118,000 . The results are grid independent.

The governing equation used is unsteady incompressible, staggered flow discretized by the finite volume method. The transient term is discretized using a second-order implicit scheme with a time step of $1 \mathrm{~s}$ and inner iteration of 20 between time steps. A second-order upwind differencing scheme was used for the convection term and a central diffusion scheme for the diffusion term. A standard $\kappa-\varepsilon$ turbulent model was used along with the SIMPLE algorithm to deal with the pressure-velocity coupling. Star $\mathrm{CCM}+7.0$ commercial software was used for the simulation, while Hypermesh 7.0 student's license for pre-processing and generating the mesh. Star CCM+ software is widely used in the automotive industry [13].

\section{EXPERIMENTS}

\subsection{Experimental Conditions}

For the validation of the results, experiments were conducted inside the cabin of a Maruti Suzuki Alto 800 , i.e. a passenger car. The parameters measured were temperature at various sample points and air-inlet temperature. Portable anemometer and thermocouples were used to measure air velocity and temperatures, respectively. The experiments were carried out at noon with an ambient temperature of $38{ }^{\circ} \mathrm{C}$ and relative humidity of $15 \%$. The test was performed after the car had reached a steady state standing in a parking environment with the sun directly overhead $(\alpha=0)$, irradiation $=1 \mathrm{~kW} / \mathrm{m}^{2}$. The experiments were repeated over a few days to investigate the repeatability and variability in measurement conditions. We observed an approximate $4 \%$ difference in the results due to variation in climatic conditions. The total time of the experiment was 10 minutes. This was to study the fastest mode of cooling a cabin that has reached a steady state while standing idle in an parking area as already discussed in the problem statement. Since the duration of experimentation was small, the outside conditions can be taken as constant during the test.

\subsection{Validation with Experimental Data}

The collected data from the various sample points are compared to the predicted values obtained using the CFD model. The cabin space is divided into four parts by the planes $z=0 \mathrm{~m}$ and $x=1.5 \mathrm{~m}$ and named as driver, passenger and rear passengers. Fig. 2 shows the sampling points for validation for each passenger, 3 for the driver and front passenger, one each at head, knee and foot region. For the back passengers 2 points were under consideration, one each at the head and foot region. The names of these points have been assigned based on the division of space and will be referred by the same names in further discussion. The average wind speed was $13 \mathrm{~km} / \mathrm{h}$ during the experimentation. The sampling rate was set to 10 seconds.

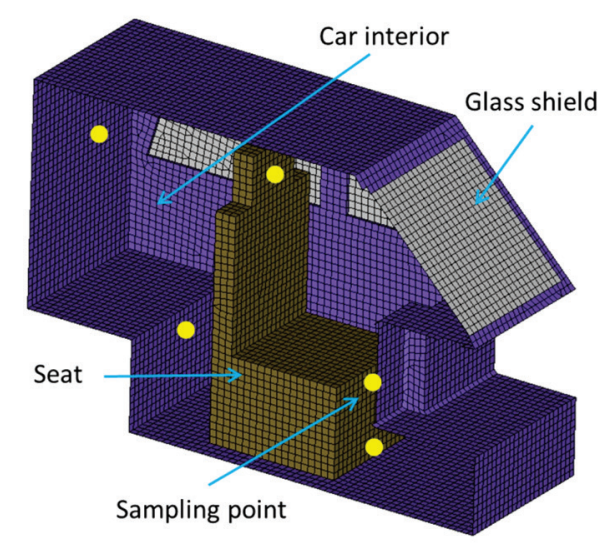

Fig. 2. Half-section view of the cabin showing the sampling points marked with yellow dots for validation with experiments

The initial temperature inside the cabin was found to be $43{ }^{\circ} \mathrm{C}$ which was mainly due to the greenhouse effect taking place inside the cabin. The air-inlet temperature was $20^{\circ} \mathrm{C}$ and the air conditioner was working in external circulation mode with a high flow setting. Fig. 3 shows the comparison between the results predicted from the CFD model and the measured temperature. It can be seen that trends in temperature variation are well captured by the software. Furthermore, the maximum difference between the predicted and measured temperature is less than $10 \%$, which is acceptable. In order to improve the accuracy of the data, four thermocouples were used at each of the sample point and an average of the input was taken. The air-inlet velocity was measured using a Testo hot-wire anemometer whose 


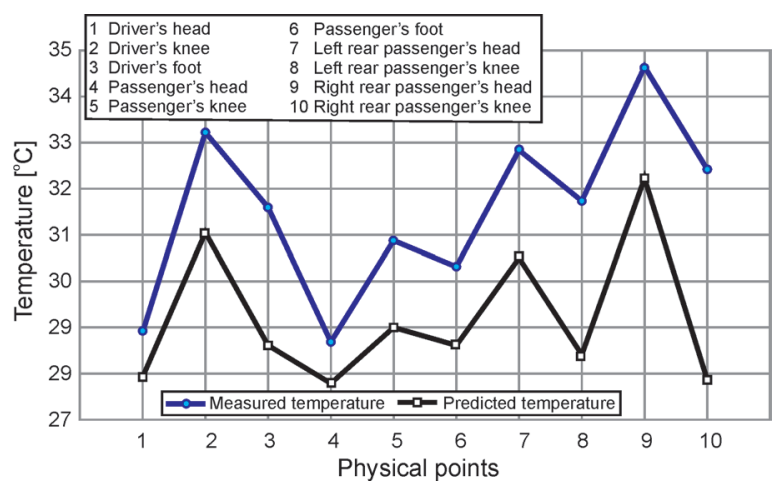

Fig. 3. Comparision with predicted temperature from CFD model and experimental data

range and accuracy were as follows: velocity range: 0 to $20 \mathrm{~m} / \mathrm{s}$, air velocity resolution: $\pm 0.01 \mathrm{~m} / \mathrm{s}$, air velocity accuracy: $\pm 0.3 \mathrm{~m} / \mathrm{s}, \pm 5 \% \mathrm{mV}$, temperature resolution: $0.1{ }^{\circ} \mathrm{C}$, temperature range: $-20{ }^{\circ} \mathrm{C}$ to $71{ }^{\circ} \mathrm{C}$, temperature accuracy: $\pm 0.5^{\circ} \mathrm{C}$. The vents were set at different flow settings such as high, medium and low air velocity. The anemometer was placed directly in front the vents. This multi-direction air velocity measuring anemometer features both timed mean (mean value calculated over a user-specified time) and multipoint mean (mean value calculated over user-entered point measurements) to help track variable flows and cyclical events. We used the timed mean air velocity measured every 5 seconds. There was no velocity fluctuation in the measured inlet vent velocity as engine load and speed were constant. Fig. 4 shows the temperature variation at various locations (see legend) with time. Note that the temperature fluctuates with time and reaches a steady case after $240 \mathrm{~s}$. These temperatures correlate well the measured temperatures to within ten percent accuracy as shown in Fig. 3. It is to be mentioned here that the results presented are in terms of cabin temperature and not on a thermal sensation scale as recommended by ASHRE standards [14] and [15]. One reason being that the industry generally relies on direct temperature measurement and later interpret it on a thermal sensation scale. While the thermal index for comfort is useful, a temperature scale serves as an important input for industry to perform various design changes.

\section{RESULTS AND DISCUSSIONS}

\subsection{Effect of Different Flow Variables}

As discussed earlier, different researchers have attempted to simulate and obtain a homogenous temperature distribution inside the car. Most of them tried to mix and match different variables, some accounting for the car body color, others testing the flow speeds and location of vents. Few took account of the role of passengers as a heat source and also reduced the effect of radiation with reflection coating. In our case we first studied the effect of air-flow positioned at different angles. Based on the results, the best possible angles were then selected and a dynamic flow inlets model was developed where the angles of the inlets were kept periodically oscillating between the chosen angles. The different attempts and their results are discussed below.

\subsubsection{Medium Flow at Zero, 30 Degrees, 45 Degrees and 60 Degrees Positive Form the Horizontal}

Medium flow means that the center inlets deliver at $4.5 \mathrm{~m} / \mathrm{s}$ and side inlets at $2 \mathrm{~m} / \mathrm{s}$ velocity. The

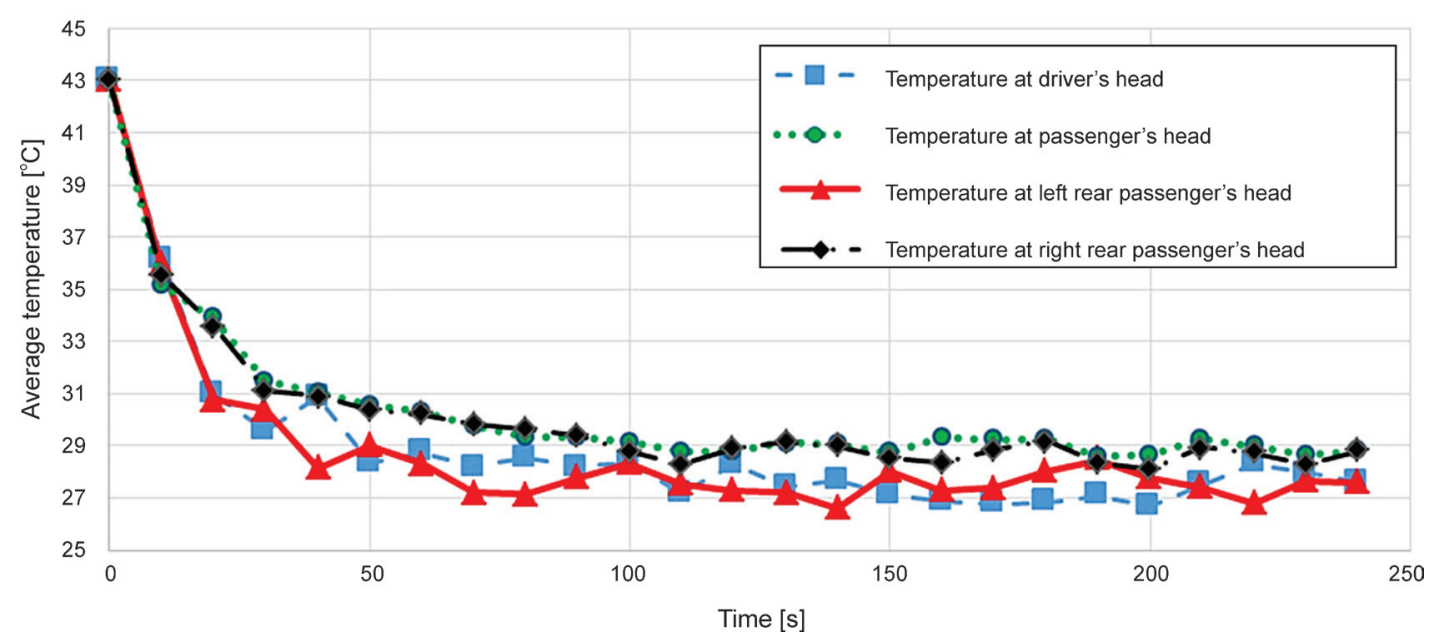

Fig. 4. Temperature variation with time at different locations 


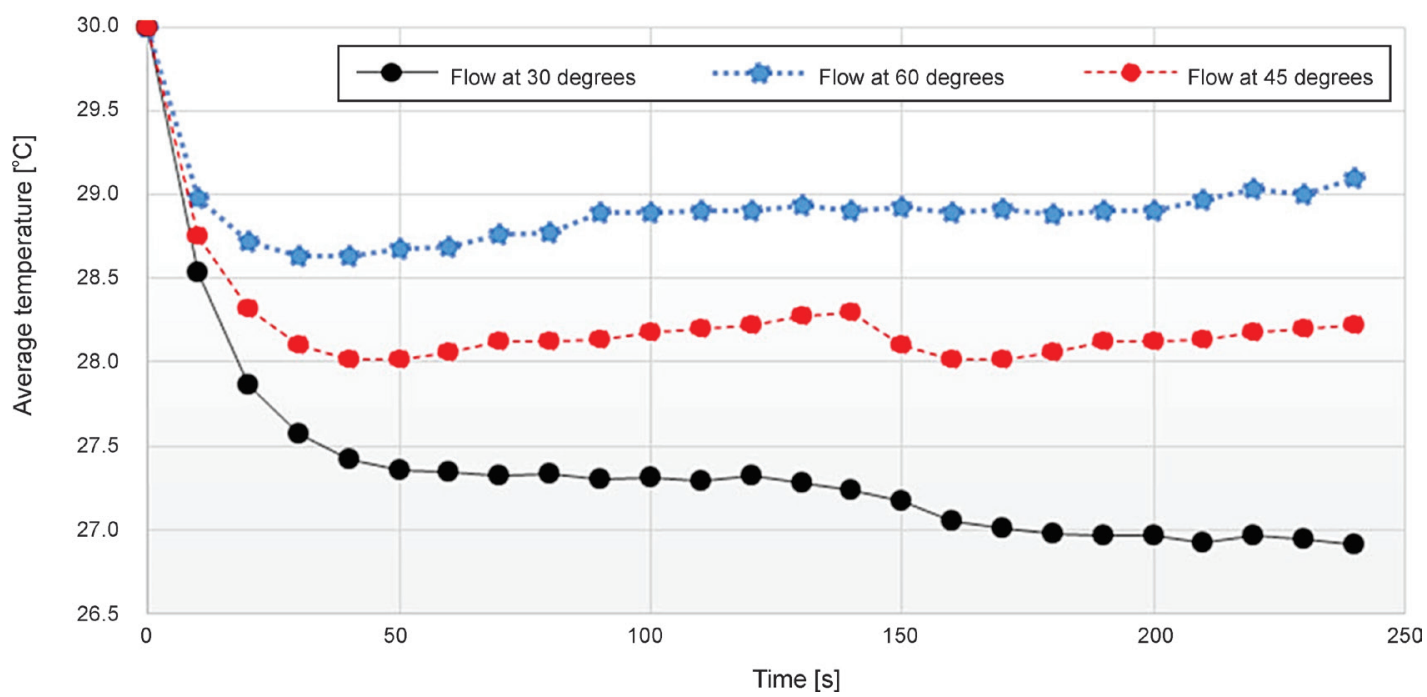

Fig. 5. Plot of average temperature vs. time for flow inlets at 30 degree, 45 degrees and 60 degrees

exterior conditions were measured to be $24{ }^{\circ} \mathrm{C}$ ambient temperature, $20 \%$ humidity and an average wind velocity of $13 \mathrm{~km} / \mathrm{h}$. The cabin steady state temperature was $30^{\circ} \mathrm{C}$. Here the angles mentioned are measured positively from the horizontal. The simulation time was four minutes with a time step of $10 \mathrm{~s}$. Hence the whole simulation was done in $240 \mathrm{~s}$ or $4 \mathrm{~min}$. This is because in order to cool the car one needs to do the maximum work in the initial phase, which will be in the first $3 \mathrm{~min}$ to $4 \mathrm{~min}$.

Comparing the plots of volume average temperature inside the computational domain vs. time (Fig. 5), it is observed that the vent flow is able to cool the cabin area when angled at 30 degrees. A drop of 3 degrees was obtained during the first 4 minutes. Although the results are comparable with the case of zero degrees it was also observed that the flow at 30 degrees seems to cool the front seats better than other three cases. Flow at 60 degrees also affects the cooling but marginally, as temperature drop was only $1{ }^{\circ} \mathrm{C}$.

\subsubsection{High Flow at Zero, 30 Degrees, 45 Degrees and 60 Degrees Positive Form the Horizontal}

All the operating conditions mentioned in Section 3.1.1 remain the same, only the flow speed is varied in this case. High flow means the center inlets deliver cooling airflow at $6.3 \mathrm{~m} / \mathrm{s}$ and the side inlets at $3 \mathrm{~m} / \mathrm{s}$.

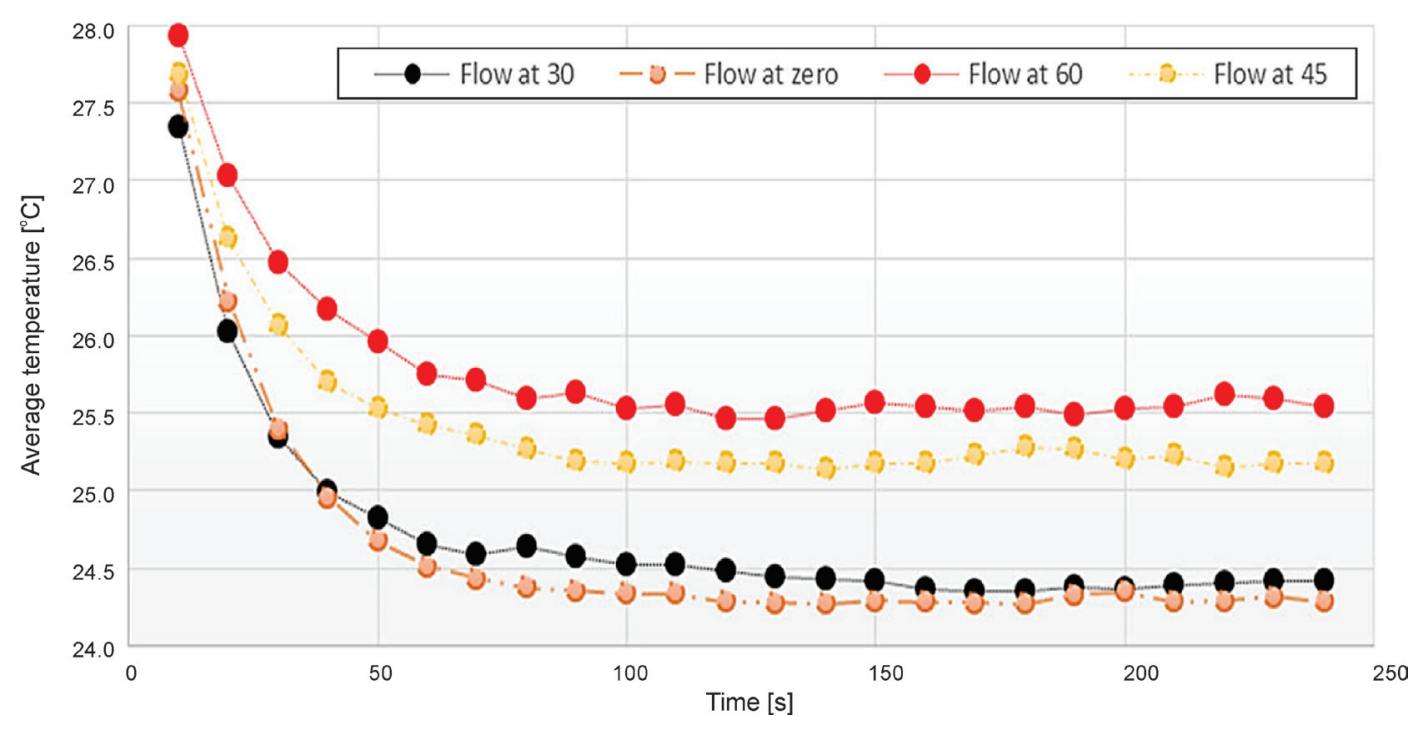

Fig. 6. Plot of Average temperature vs. time for flow inlets at zero degree, 30 degree, 45 degrees and 60 
The plots depict similar results as predicted earlier with the airflow at 30 degrees. The only change is that the temperature drops obtained in this case have increased, as shown in Fig. 6.

The velocity vector plot and the temperature scalar plots also suggest the same phenomenon. The air-flow circulation phenomena observed earlier can also be seen in the high flow case (Figs. 7 and 8). When positioned at 30 degrees the air inlets are now able to cool the front region to 23 degrees and the average temperature drops by $6{ }^{\circ} \mathrm{C}$. Although the objective to cool the cabin has been achieved, non-homogeneity of temperature still remains a challenge. Similarly, when vanes angles are at 60 degrees the inlets are not able to cool the front as effectively as with 30 degrees. However, since the flow rates are higher the average temperature now drops by 5 degrees. Vane angles of 45 degrees performs only slightly better than those at 60.

\subsection{Effect of Dynamic Air-Inlets}

From the earlier discussion in Sections 3.1.1 and 3.1.2 it is clear that each angle is successful in cooling one or the other region at about the $X=1.5 \mathrm{~m}$ plane. Flow at 30 degrees cools the front half and achieves the maximum drop in temperature, flow at 60 degrees manages to cool the rear slightly, whereas flow at 45
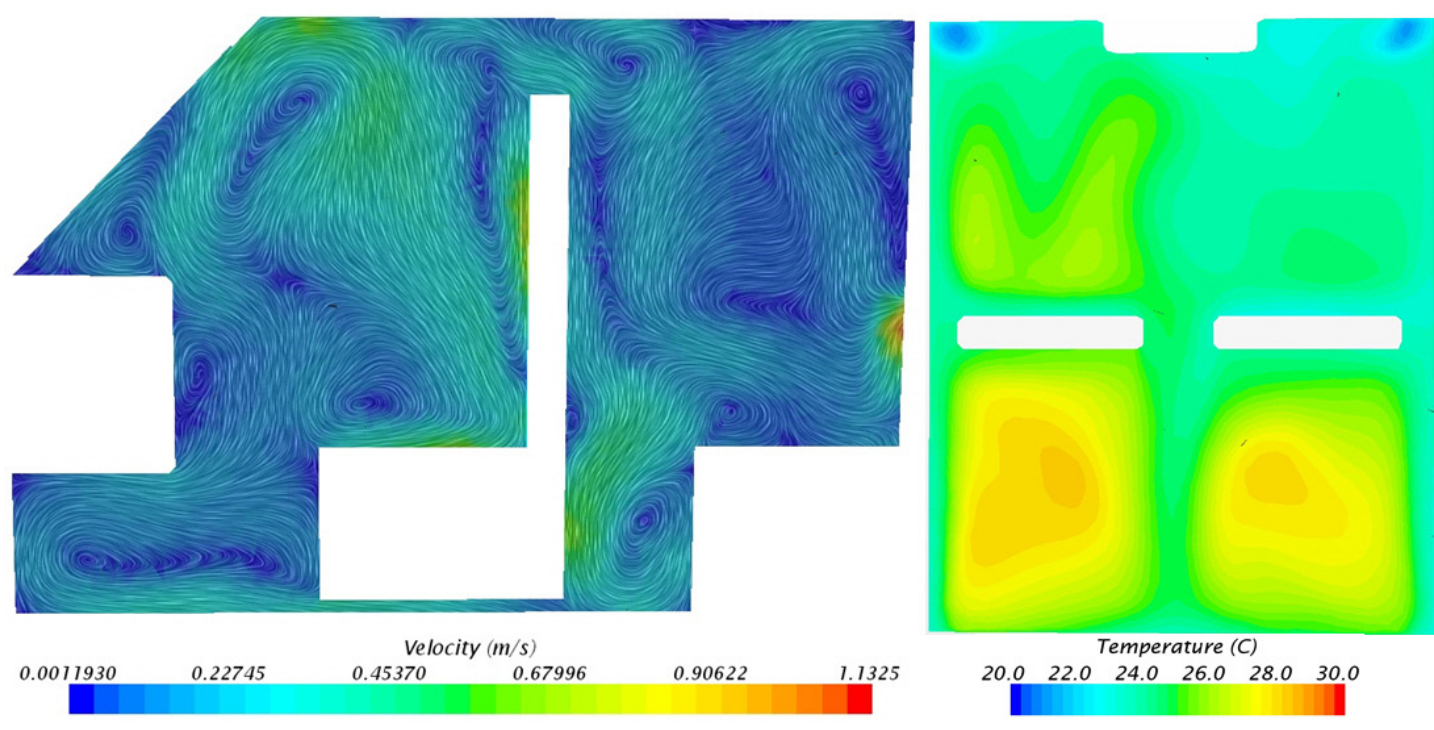

Fig. 7. High flow at a flow angle of 30 degrees. Left: velocity vector and, right: temperature contours
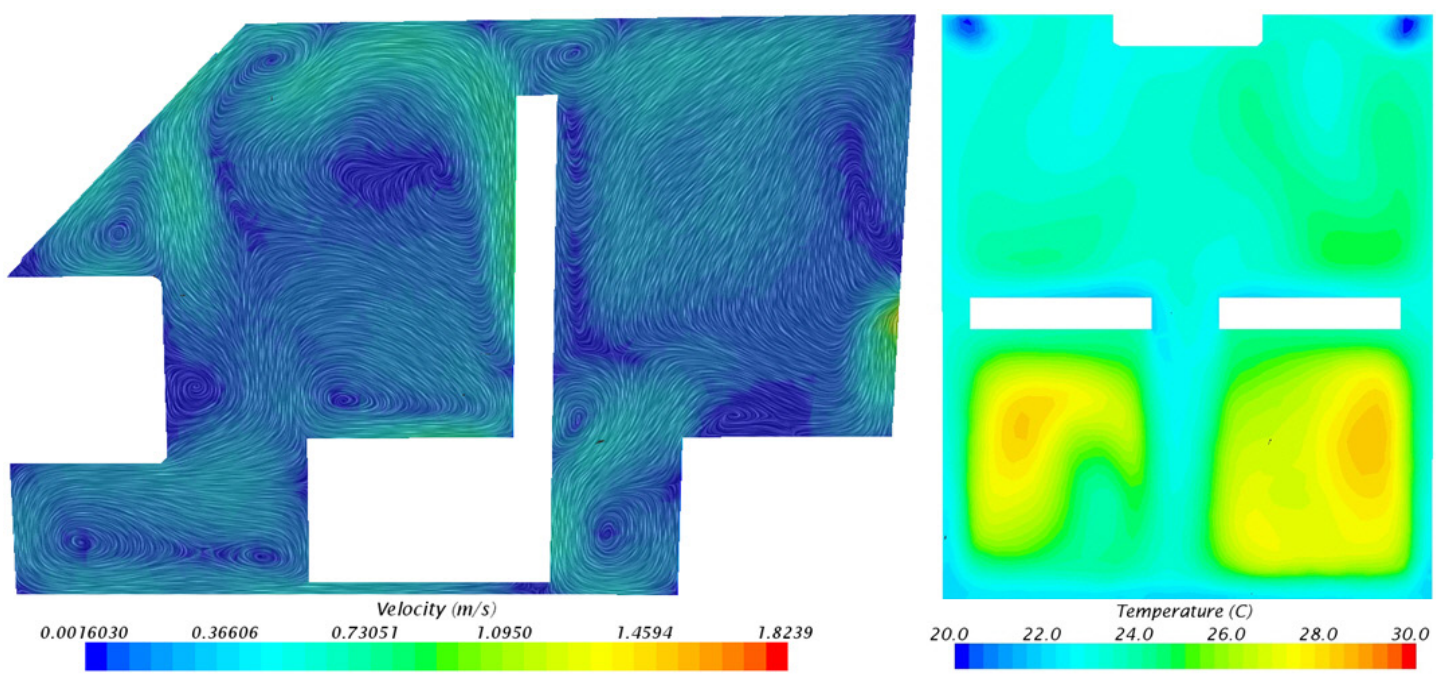

Fig. 8. High flow at a flow angle of 60 degrees 
degrees creates a homogenous temperature drop. The circulation keeps the heated air in the same region as in the case of 60 degrees. Thus the heated air has to be continuously replaced and re-circulation should be used wisely.

Thus to study the effect of all these, the concept of dynamic vents was attempted next and simulated in the computational domain. Dynamic vents describe air-inlet vents that swing automatically between the chosen flow angles at some set frequency. This way the vents can cover all the angles from 30 degrees to 45 degrees and then 45 degrees to 60 degrees. Accordingly, changes in angles and frequency for different cases have been made and discussed further.

\subsubsection{Dynamic Vents with Flow Angles Covering Zero to $\mathbf{3 0}$ Degrees with Medium Flow Setting}

In this case the vents go from zero to 30 degrees in 8 seconds. The flow rate is set at a medium setting and other conditions remain the same as in Section 3.1. The simulation time is $4 \mathrm{~min}$. It was observed that dynamic vents have been successful in cooling the rear area with a minimum temperature of 24.5 ${ }^{\circ} \mathrm{C}$. They have also performed better in maintaining a uniform distribution. Nevertheless, the average temperature is still recorded to be $26^{\circ} \mathrm{C}$ (Fig. 9 shows temperature at $x=1.5 \mathrm{~m}$ ). Thus the test with dynamic vents has shown that uniform temperature distribution is possible, which has encouraged us to make further simulations with different variable angles.

\subsubsection{Dynamic Vents with Flow Angles Covering Zero to $\mathbf{3 0}$ Degrees with High Flow Setting}

In this case, the high flow cooled the cabin by 6 degrees but the effect cannot be ascribed to the dynamic vents at a medium flow setting. The temperature drop attained is mainly due to the increased flow rate.

The above discussion demonstrates that the use of dynamic vents can achieve the desired uniformity. In Section 3.1 and sub-sections it was concluded that a flow angle of 30 degrees cools the front and 60 degrees cools the back whereas 45 degrees lies in between. Hence, we shall now try to use the results of both stationary and dynamic vents, thereby resulting in further tests where the vents will now traverse 30 degrees to 60 degrees at different frequencies.

\subsection{Dynamic Vents with Flow Angles Covering 30 Degrees to 60 Degrees}

As discussed earlier, the whole cabin should be cooled as the vents will traverse all the angles earlier investigated. It was observed that for both medium and high flow rates the cooling effects are similar. The high flow cools the cabin interiors at a fast rate with a drop of $6.7^{\circ} \mathrm{C}$.

The temperature distribution is also uniform in the high flow case, as seen in the former case. Thus, the results cannot be concretely credited to the dynamic vents. Next we try to vary the frequencies of traversing the angle so as to arrive at more concrete conclusions.

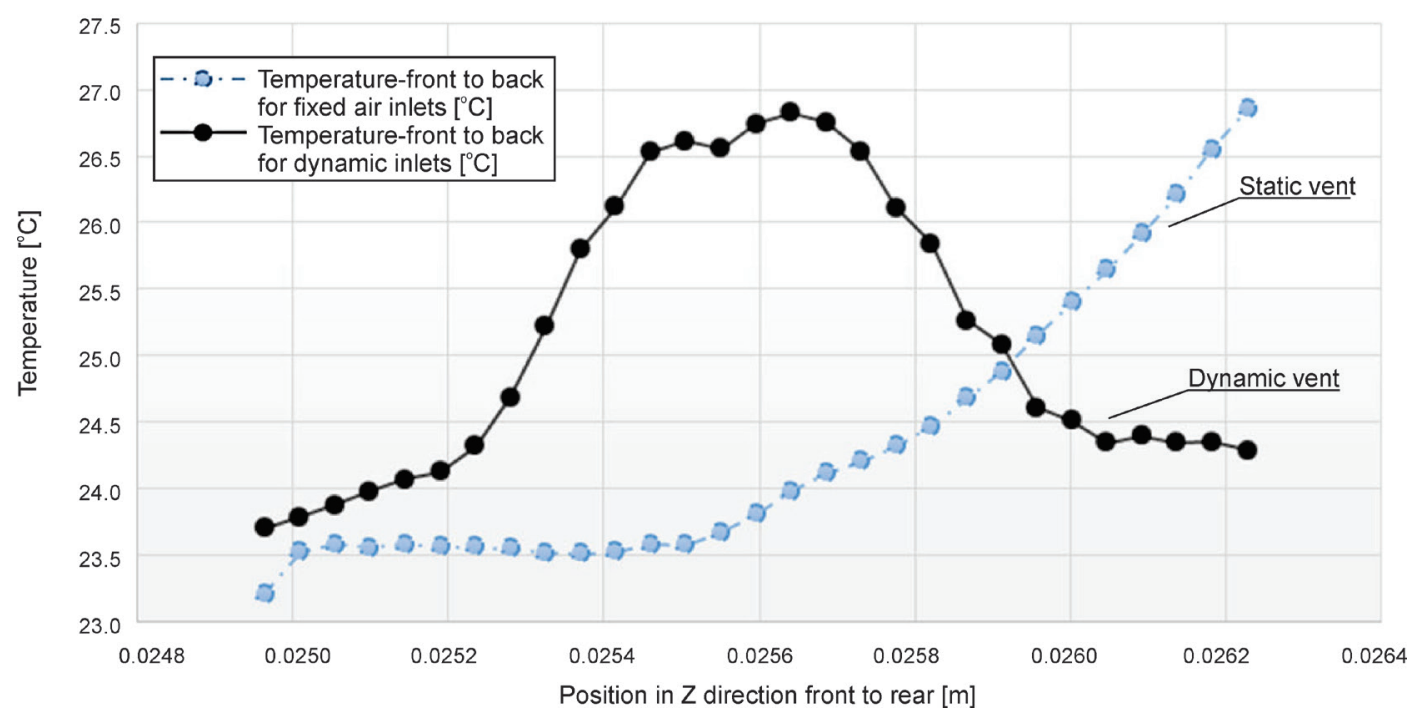

Fig. 9. Comparison of X-Y Plot of average temperature from front to back on a horizontal plane between stationary and dynamic vents 


\subsection{Dynamic Vents with Flow Angles Covering 30 Degrees to 60 Degrees in 6 Seconds}

All the external conditions are similar to those in the earlier cases and no changes have been made to the vents, only the frequency at which the vents sweep has been changed. The vents now traverse the angles 30 to 60 periodically in $6 \mathrm{~s}$ and back. Comparing the temperature contours (Fig. 10 and 11) for both the medium and high flow settings it is observed that both of these settings are able to provide a uniform temperature distribution. The average temperature for a medium setting is $25.8^{\circ} \mathrm{C}$, whereas for the high flow setting the average temperature drops to $23.6{ }^{\circ} \mathrm{C}$. Thus, changing the frequency has resulted in dropping the temperature inside the cabin as well as maintaining uniformity.

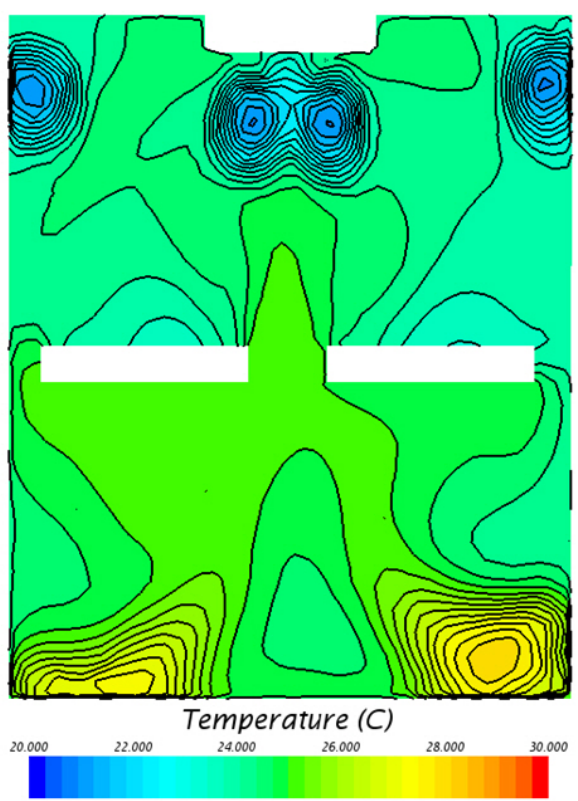

Fig. 10. Top view of temperature contour with dynamic vents traversing 30 degrees to 60 degrees in $6 \mathrm{~s}$ at a medium flow setting

By further increasing the frequency, where the half time period is $4 \mathrm{~s}$, no significant change is observed and the results are quite similar. Thus, any frequency whose time period is below $12 \mathrm{~s}$ is able to generate the desired effect, but due to constraints on the efficiency of such mechanical systems the optimum frequency would be when the time period is $12 \mathrm{~s}$. Results are summarized in Table 1.

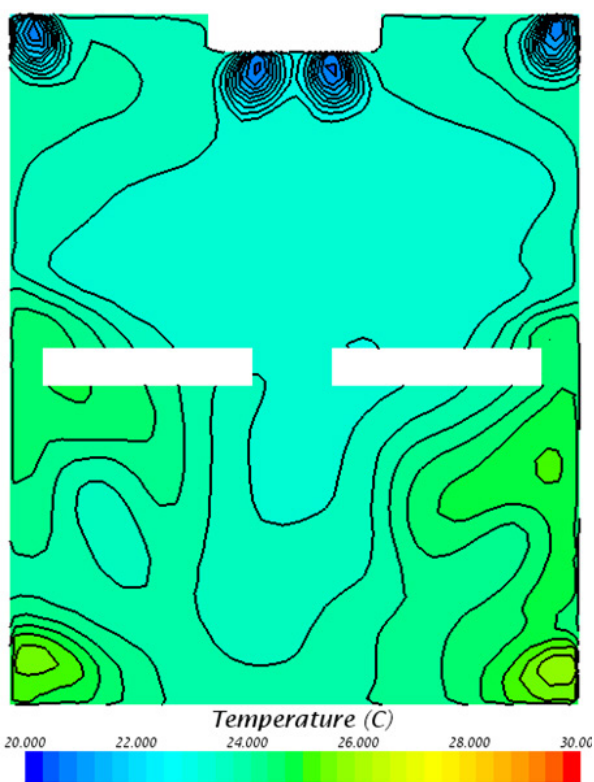

Fig. 11. Top view of temperature contour with dynamic vents traversing 30 degrees to 60 degrees in $6 \mathrm{~s}$ using a high flow setting

Table 1. Summary of volume averaged temperature drop at various angles; the highest temperature drop was observed for dynamic vents for 30 degrees to 60 degrees

\begin{tabular}{ccc}
\hline Cases & $\begin{array}{c}\text { Temperature drop } \\
\text { (Angles) }\end{array}$ & $\begin{array}{c}\text { Temperature drop } \\
\text { (Angle Sweep) }\end{array}$ \\
\hline \multirow{2}{*}{ Static vents } & $5.75(0), 5.52(30)$, & \\
& $4.75(45), 4.50(60)$ & \\
Dynamic vents & & $6(0$ to 30$)$, \\
& & $6.7(30$ to 60$)$ \\
\hline
\end{tabular}

\section{CONCLUSIONS}

It is evident that the maximum temperature drop is obtained when the vent angle is 30 degrees. The average temperature in this case was $26.8{ }^{\circ} \mathrm{C}$ at a medium setting and $24.45{ }^{\circ} \mathrm{C}$ at a high flow setting after $4 \mathrm{~min}$. However, the temperature distribution was not homogenous and the vents were unable to cool the rear area.

A further drop was obtained in the case of dynamic vents sweeping angles from 30 degrees to 60 degrees in $6 \mathrm{~s}$. The average temperature in this case was $25.8{ }^{\circ} \mathrm{C}$ at a medium flow setting and $23.6^{\circ} \mathrm{C}$ at a high flow setting after 4 minutes from the start and, thus contributing to an extra drop of $1{ }^{\circ} \mathrm{C}$ in both high and medium settings as compared to static vent analysis. Additionally the temperature distribution obtained is uniform throughout the cabin space. Hence it is proposed on the basis of the results that for the 
first three to four minutes the air-conditioner should be operated at a high flow setting using the dynamic vents as discussed earlier. Then the regular mode of operation can be used. An eccentric rotating shaft can be used to convert static vents into dynamic vents. Home air-conditioners use dynamic vents to achieve quicker cooling.

All these results show the scope for future study where changes can be made to the positions of the vents. Additional air-inlets can be incorporated on the roof top for the rear passengers since this is a difficult area to cool. The effect of changing the flow rate by using a more powerful air-conditional system can also be studied. Studies have already been carried out to provide a particular passenger with a particular temperature setting. This can be achieved through the use of spot-cooling using nozzles, which is an emerging area of research in the field of passenger comfort. Moreover the effect of passengers and their body heat can also be included in the simulations and make the system closer to a real life situation.

However, it should be noted that any modifications to the air-conditioning system would put an additional burden on the engine and thus affect the efficiency of the engine along with increasing the cost of running the car. Hence, a compromise has to be made while selecting the variables for optimum efficiency... and comfort.

\section{REFERENCES}

[1] ANSI/ASHRAE Standard 55-2013, Thermal Environmental Conditions for Human Occupancy. Approved American National Standard, Atlanta.

[2] Musat, R., Helerea, E. (2009). Parameters and models of the vehicle thermal comfort. Acta Universitatis Sapientiae, Electrical and Mechanical Engineering, vol. 1, p. 215-226.

[3] Chen, K.H., Kaushik, S., Han, T., Ghosh, D., Wang, M. (2012). Thermal comfort prediction and validation in a realistic vehicle thermal environment. SAE Technical Paper, 2012-01-0645, D0l:10.4271/2012-01-0645.

[4] Limaye, V.M., Deshpande M.D., Sivapragasam, M., Kumar, V. (2012). Design of dynamic airvents and airflow analysis in a passenger car cabin. SASTech - Technical Journal, vol. 11, no. 1, p. $41-48$.

[5] Walgama, C., Fackrell, S., Karimi, M., Fartaj, A., Rankin, G.W. (2006). Passenger thermal comfort in vehicles-a review. Proceedings of the Institution of Mechanical Engineers, Part D: Journal of Automobile Engineering, vol. 220, no. 5, p. 543562, D0I:10.1243/09544070D00705.

[6] Zhang, H.-J., Dai, L., Xu, G.-Q., Li, Y., Chen, W., Tao, W.-Q. (2009). Studies of air-flow and temperature fields inside a passenger compartment for improving thermal comfort and saving energy, Part I: Test/numerical model and validation. Applied Thermal Engineering, vol. 29, no. 10, p. 2022-2027, DOI:10.1016/j.applthermaleng.2008.10.005.

[7] Ruzic, D. (2011). Improvement of thermal comfort in a passenger car by localized air distribution. Acta Technical Corviniesis - Bulletin of Engineering, vol. 4, no. 1, p. 63-67.

[8] Alexandrov, A., Kudriavtsev, V., Reggio, M. (2001). Analysis of flow patterns and heat transfer in generic passenger car mini-environment. 9th Annual Conference of CFD Society of Canada, Ontario, p. 27-29.

[9] Wan, J.W., van der Kooi, J. (1991). Influence of the position of supply and exhaust openings on comfort in a passenger vehicle. International Journal of Vehicle Design, vol. 12, no. 5-6, p. 588-597, D0I:10.1504/IJVD.1991.061702.

[10] Zvar Baskovic, U., Lorenz, M., Butala, V. (2014). Adiabatic flow simulation in an air-conditioned vehicle passenger compartment. International Journal of Simulation Modelling, vol. 13, no. 1, p. 42-53, D0I:10.2507/IJSIMM13(1)4.253.

[11] Ismail, A.R., Karagaratnan, S.K., Kadirgama, K. (2013). Thermal comfort findings: scenario at Malaysian automotive industry. Thermal Science, vol. 17, no. 2, p. 387-396, DOI:10.2298/TSCI111111015I.

[12] Muhammad lqbal (2012). An Introduction to Solar Radiation, 1st ed., Elsevier, New York..

[13] Ahmad, N. E., Abo-Serie, E., Gaylard, A. (2010). Mesh optimization for ground vehicle aerodynamics. CFD Letters, vol. 2, no. 1, p. 54-65.

[14] Attia, A., EL Helw, M., Teamah, H. (2013). Three-dimensional thermal comfort analysis for hospital operating room with the effect of door gradually opened, Part (II) Effect on mean age of the air and predicted mean vote distribution. CFD Letters, vol. 5, no. 2, p. 20-31.

[15] Attia, A., EL Helw, M., Teamah, H. (2013). Three-dimensional thermal comfort analysis for hospital operating room with the effect of door gradually opened, Part (I) Effect on velocity and temperature distributions. CFD Letters, vol. 5, no. 1-2, p. 6-19. 